\title{
Instantaneous Dynamics of QCD
}

\author{
Patrick Cooper ${ }^{1, a}$ and Daniel Zwanziger 2 ,b, c \\ ${ }^{1}$ Physics Department, Duquesne University, 600 Forbes Ave, Pittsburgh, PA 15282, USA \\ ${ }^{2}$ Physics Department, New York University, 4 Washington Place, New York, NY 10003, USA
}

\begin{abstract}
We start from the observation that, in the confining phase of QCD, the instantaneous color-Coulomb potential in Coulomb gauge is confining. This suggests that, in the confining phase, the dynamics, as expressed in the set of Schwinger-Dyson equations, may be dominated by the purely instantaneous terms. We develop a calculational scheme that expresses the instantaneous dynamics in the local formulation of QCD that includes a cut-off at the Gribov horizon. ${ }^{\mathrm{d}}$
\end{abstract}

\section{Introduction}

In sections 1 through 9 we outline the properties and derivation of the instantaneous dynamics expressed in the instantaneous Schwinger-Dyson equations (ISDE). In sect. 10, we write the ISDE in diagrammatic form.

\section{No confinement without Coulomb confinement}

Gribov's insight into the mechanism of confinement [1] is substantiated by the theorem [2],

$$
V_{\text {coul }}(R) \geq V_{\text {wilson }}(R)
$$

for $R \rightarrow \infty$, where the color-Coulomb potential, $V_{\text {coul }}(R)$, is the instantaneous part of the zero-zero component of the gluon propagator in Coulomb gauge, ${ }^{1}$

$$
g^{2} D_{00}(R, T)=V_{\text {coul }}(R) \delta(T)+\text { non }- \text { instantaneous },
$$

and $V_{\text {wilson }}(R)$ is the gauge-invariant potential derived from a large rectangular Wilson loop. Accordingly, when $V_{\text {wilson }}(R)$ rises linearly, $V_{\text {coul }}(R)$ rises linearly or super-linearly.

\footnotetext{
ae-mail: cooperp@duq.edu

be-mail: dz2@nyu.edu

${ }^{\mathrm{c}}$ Speaker, XIIth Quark Confinement and the Hadron Spectrum, 28 August to 4 September, 2016, Thessaloniki, Greece

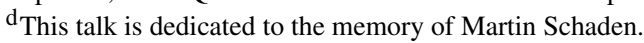

${ }^{1}$ It is shown in [3] that $V_{\text {coul }}(R)$ and $g A_{0}$ are renormalization-group invariants in Coulomb gauge.
} 


\section{Motive and method of present approach}

Motivated by this theorem, we conjecture that, in the Coulomb gauge, the instantaneous dynamics is dominant. This will be expressed in a closed system of instantaneous Schwinger-Dyson equations (ISDE).

We shall work within the framework of local quantum field theory, with a local action that encodes the cut-off at the Gribov horizon [4], and moreover that is BRST-invariant, thus preserving the geometric property of a gauge theory. This action is derived by the method of Maggiore-Schaden [5] in which BRST-symmetry is spontaneously broken [6]. A conjecture is offered in [7,8] for the identification of physical operators and the physical subspace, but this important subject will not be discussed here.

We shall derive the ISDE by extending to the local action that enforces the cut-off at the Gribov horizon the method which was previously applied to the Faddeev-Popov action [9].

\section{Horizon function and non-local action}

We shall localize the non-local Euclidean action,

$$
S=S_{\mathrm{FP}}+\gamma H-\gamma \int d^{d} x d\left(N^{2}-1\right)
$$

where $S_{\mathrm{FP}}$ is the (local) Faddeev-Popov action in Coulomb gauge, $H$ is the non-local horizon function given by

$$
H \equiv \int d^{d} x d^{d} y D_{\mu}^{a b}(x) D_{\mu}^{a c}(y)\left(M^{-1}\right)^{b c}(x, y ; A),
$$

and $\gamma^{1 / 4}$ is the Gribov mass. $H$ cuts off the functional integral at the Gribov horizon, as one sees from the eigenfunction expansion,

$$
\left(M^{-1}\right)^{b c}(x, y ; A)=\sum_{n} \frac{\psi_{n}^{b}(x) \psi_{n}^{c}(y)}{\lambda_{n}(A)},
$$

where the $\lambda_{n}(A)$ are the eigenvalues of the Faddeev-Popov operator $M \equiv-\nabla \cdot \mathbf{D}(\mathbf{A})$ in Coulomb gauge. The lowest non-trivial eigenvalue $\lambda_{1}(A)$ approaches 0 as the Gribov horizon is approached from within.

\section{Horizon condition and Kugo-Ojima confinement condition}

The Gribov mass $\gamma^{1 / 4}$ is not an independent parameter, but is fixed by the horizon condition

$$
<H>=d\left(N^{2}-1\right) \int d^{d} x .
$$

It is a remarkable fact that the horizon condition and the famous Kugo-Ojima confinement condition $[10,11]$ are the same statement

$$
-i \int d^{d} x<\left(D_{\mu} c\right)^{a}(x)\left(D_{\mu} \bar{c}\right)^{a}(0)>=d\left(N^{2}-1\right)
$$

This may indicate that color confinement is assured in this theory, although the precise hypotheses of the Kugo-Ojima theorem are not satisfied in this approach. 


\section{Horizon condition and dual Meissner effect}

It is also a remarkable fact that the horizon condition is equivalent to the statement that the QCD vacuum is a perfect color-electric superconductor, which is the dual Meissner effect [12],

$$
\begin{gathered}
G(\vec{k})=\frac{d(\vec{k})}{\vec{k}^{2}}=\frac{1}{\epsilon(\vec{k}) \vec{k}^{2}} \\
d^{-1}(\vec{k}=0)=0 \Longleftrightarrow \epsilon(\vec{k}=0),
\end{gathered}
$$

where $G(\vec{k})$ is the ghost propagator and $\epsilon(\vec{k})$ is the dielectric constant.

\section{Auxiliary ghosts}

Just as the Faddeev-Popov determinant is localized by introducing ghosts,

$$
\operatorname{det} M=\int d c d \bar{c} \exp \left(-\int d^{d} x \bar{c} M c\right),
$$

likewise, the horizon function $H$ may be localized by introducing "auxiliary" ghosts [4],

$$
\exp (-\gamma H)=\int d \varphi d \bar{\varphi} d \omega d \bar{\omega} \exp \left(-\int d^{d} x\left[\bar{\varphi} M \varphi-\bar{\omega} M \omega+\gamma^{1 / 2} D \cdot(\varphi-\bar{\varphi})\right]\right) .
$$

For reviews of this approach, see [13, 14].

\section{Cancellation of energy divergences}

The Coulomb gauge is plagued by energy divergences. For example, the integrand of the ghost loop is independent of $p_{0}$,

$$
\int d^{3} p d p_{0} \frac{1}{\vec{p}^{2}} \frac{1}{(\vec{p}-\vec{k})^{2}}
$$

which leads to the horrible energy divergence

$$
\int d p_{0} 1=\infty
$$

We get rid of such divergences by using the first-order formalism in which they cancel manifestly $[3,15]$. This relies on the fact that the Coulomb gauge is a unitary gauge. The first-order action is obtained by writing

$$
\exp \left(-\int d^{4} x F_{0 i}^{2} / 2\right)=\int d^{3} \pi \exp \left(\int d^{4} x\left(i \pi_{i} F_{0 i}-\pi^{2} / 2\right)\right)
$$

Here $F_{0 i}=\partial_{0} A_{i}-D_{i} A_{0}$, the Coulomb gauge condition $\partial_{i} A_{i}=0$ holds identically, and $\pi_{i}$ is colorelectric field. It is decomposed into its transverse and longitudinal parts,

$$
\pi_{i}=\tau_{i}-\partial_{i} \lambda
$$

with $\partial_{i} \tau_{i}=0$. As a result of the equality (23), given below, the $c-\bar{c}$ ghost loop cancels the $\lambda-A_{0}$ loop,

$$
\int d^{d+1} p\left[D_{A_{0} \lambda}(\vec{p}) D_{A_{0} \lambda}(\vec{k}+\vec{p})-D_{c \bar{c}}(\vec{p}) D_{c \bar{c}}(\vec{k}+\vec{p})\right]=0
$$

and likewise for the pairs of auxiliary ghosts. This cancellation eliminates the unwanted energy divergences. 


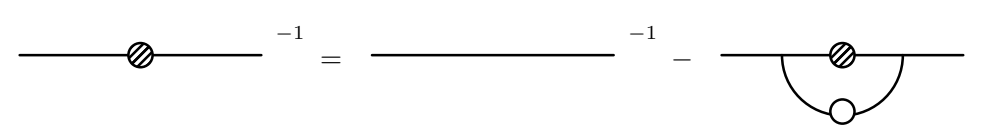

Figure 1. Schematic form of the ISDE. The shaded circle represents an instantaneous dressed propagator and the empty circle a non-instantaneous dressed propagator. All three-point vertices are tree-level.

\section{Local action and physical degrees of freedom in Coulomb gauge}

The local action is given by ${ }^{2}$

$$
\begin{gathered}
S=\int d^{d+1} x\left(\mathcal{L}_{1}+\mathcal{L}_{2}+\mathcal{L}_{3}\right) \\
\mathcal{L}_{1}=i \tau_{i} D_{0} A_{i}+(1 / 2)\left[\tau^{2}+(\partial \lambda)^{2}+B^{2}\right] \\
\mathcal{L}_{2}=i \partial_{i} \lambda D_{i} A_{0}-\partial_{i} \bar{c} D_{i} c+\partial_{i} \bar{\varphi}_{j} D_{i} \varphi_{j}-\partial_{i} \bar{\varphi}_{j} D_{i} \varphi_{j}-\partial_{i} \bar{\omega}_{j} D_{i} \omega_{j} \\
\mathcal{L}_{3}=\gamma^{1 / 2} g f^{a b c} A_{j}^{b}(\varphi-\bar{\varphi})_{j a}^{c},
\end{gathered}
$$

and $B_{i}$ is the color-magnetic field. Only the first term, $i \tau_{i} \partial_{0} A_{i}$, contains a time derivative. Because $A_{i}$ is identically transverse, $\partial_{i} A_{i}=0$ (as is $\tau_{i}$ ), $\partial_{0}$ acts on the two would-be physical degrees of freedom. The remaining terms impose constraints in the local theory. The energy divergences cancel between pairs of fermi and bose ghost loops, including the first pair, $i \partial_{i} \lambda D_{i} A_{0}-\partial_{i} \bar{c} D_{i} c$. The last term, $\mathcal{L}_{3}$, mixes bose-ghost and gluon fields.

In the following it will be convenient to change variables from $\varphi$ and $\bar{\varphi}$ to $U$ and $V$ defined by

$$
\varphi=(U+i V) / \sqrt{2} ; \quad \bar{\varphi}=(U-i V) / \sqrt{2} .
$$

The $V$-field mixes with the gluon field $A$, whereas the $U$-field does not.

The propagators of the fields $\lambda$ and $A_{0}$ are related to the ghost propagator $D_{c \bar{c}}$ by

$$
\left(\begin{array}{cl}
D_{\lambda \lambda} & D_{\lambda A_{0}} \\
D_{A_{0} \lambda} & D_{A_{0} A_{0}}
\end{array}\right)^{a b}=\delta^{a b}\left(\begin{array}{cl}
0 & -i D_{c \bar{c}}(\vec{k}) \\
-i D_{c \bar{c}}(\vec{k}) & \Gamma_{\lambda \lambda}(\vec{k}) D_{c \bar{c}}^{2}(\vec{k})
\end{array}\right),
$$

where $\Gamma_{\lambda \lambda}$ is the 2-point vertex function. The equality of the Faddeev-Popov ghost propagator and the bose-ghost propagator,

$$
\Gamma_{A_{0} \lambda}^{-1}=D_{\lambda A_{0}}=-i D_{c \bar{c}}(\vec{k}) \text {. }
$$

assures that the corresponding fermi and bose loops yield Faddeev-Popov determinants that cancel exactly,

$$
\frac{D(M)}{D(M)}=1
$$

\section{Instantaneous Schwinger-Dyson equations (ISDE)}

The truncation scheme which we use is represented schematically in Fig. 1, with detailed diagrams given in Figs. 2 and 3. Propagators such as $D_{\lambda A_{0}}$ and $D_{A V}$ represent mixing.

\footnotetext{
${ }^{2}$ For the action at finite temperature, see [16].
} 


\begin{tabular}{|c|c|c|c|c|c|}
\hline$D_{V^{P} V^{P}}^{(0)}$ & $\rightarrow$ & ---------- & $D_{A A}^{(0)}$ & $\rightarrow$ & $\sim \sim \sim \sim \sim$ \\
\hline$D_{\tau \tau}^{(0)}$ & $\rightarrow$ & $\approx \Omega \Omega \Omega \Omega \Omega$ & $D_{A_{0} A_{0}}^{(0)}$ & $\rightarrow$ & \\
\hline$D_{A V}^{(0)}$ & $\rightarrow$ & $\sim \sim---$ & $D_{\lambda A_{0}}^{(0)}$ & $\rightarrow$ & \\
\hline
\end{tabular}

Figure 2. Free propagators that appear in the ISDE. $V^{P}$ is the part of the bose-ghost propagator that mixes with the gluon propagator.

In Coulomb gauge, the propagators in general decompose into an instantaneous part and a noninstantaneous part,

$$
\begin{gathered}
D\left(\vec{x}, x_{0}\right)=D_{I}(\vec{x}) \delta\left(x_{0}\right)+D_{N}\left(\vec{x}, x_{0}\right) \\
D\left(\vec{p}, p_{0}\right)=D_{I}(\vec{p})+D_{N}\left(\vec{p}, p_{0}\right),
\end{gathered}
$$

where $\lim _{p_{0} \rightarrow \infty} D_{N}\left(\vec{p}, p_{0}\right)=0$. The ISDE is obtained as follows.

I. Cancel loops with two instantaneous bose-ghost propagators against similar fermi-ghost loops, to get rid of energy divergences, ${ }^{3}$

$$
\int d^{d+1} p\left[D_{\text {Iboson }}(\vec{p}) D_{\text {Iboson }}(\vec{k}+\vec{p})-D_{\text {Ifermion }}(\vec{p}) D_{\text {Ifermion }}(\vec{k}+\vec{p})\right]_{\text {energy divergence }}=0
$$

II. Neglect loops with two non-instantaneous propagators,

$$
\int d^{d+1} p\left[D_{N}(p) D_{N}(k+p)\right] \rightarrow 0 .
$$

III. Keep loops with one instantaneous propagator and one non-instantaneous propagator,

$$
\int d^{d+1} p\left[D_{N}\left(\vec{p}, p_{0}\right) D_{I}(\vec{k}+\vec{p})\right] .
$$

Only the equal-time part of any non-instantaneous propagator contributes to the graphs we consider,

$$
\int d^{d+1} p\left[D_{N}\left(\vec{p}, p_{0}\right) D_{I}(\vec{k}+\vec{p})\right]=\int d^{d} p\left[D^{\mathrm{ET}}(\vec{p}) D_{I}(\vec{k}+\vec{p})\right]
$$

where the equal-time part of the propagator is defined by

$$
\int d p_{0} D_{N}\left(\vec{p}, p_{0}\right)=D^{\mathrm{ET}}(\vec{p})
$$

Thus, in all graphs that we consider, the non-instantaneous propagator gets replaced by its equal-time part. The diagrams corresponding to the ISDE are given in Fig. 3.

Calculations with the ISDE will be reported elsewhere [17].

\footnotetext{
${ }^{3}$ The part of the bose-ghost loop, that is caused by mixing with the gluon propagator, survives.
} 


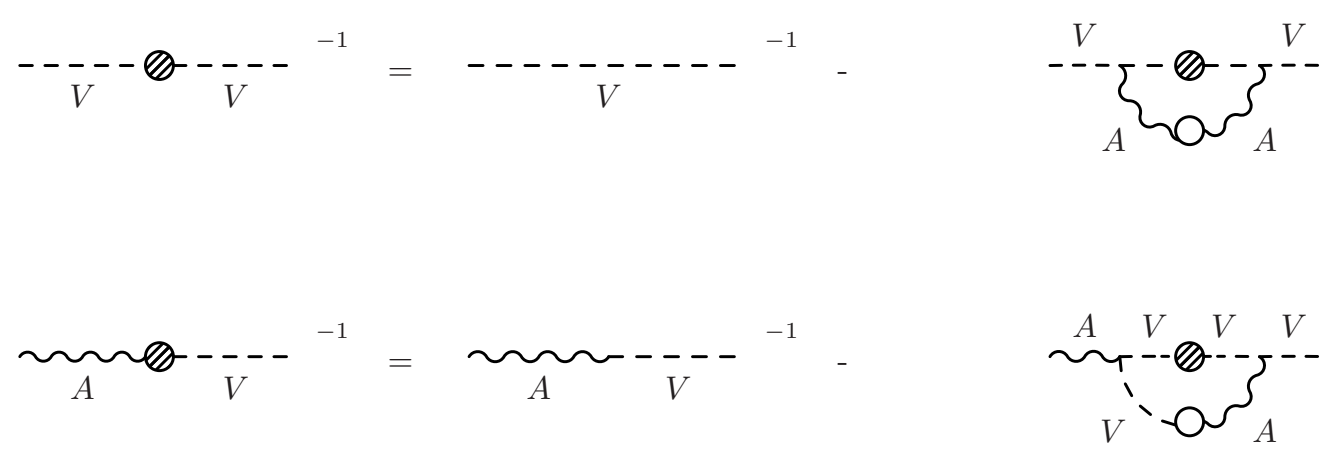

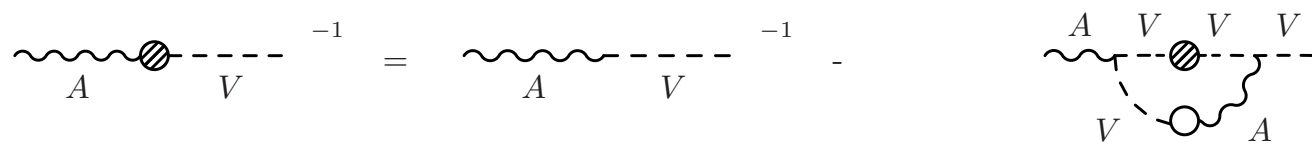

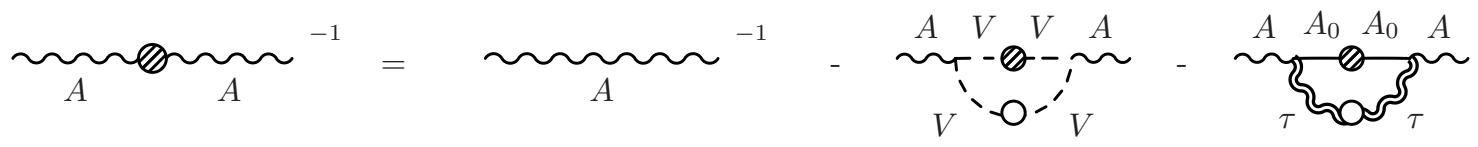
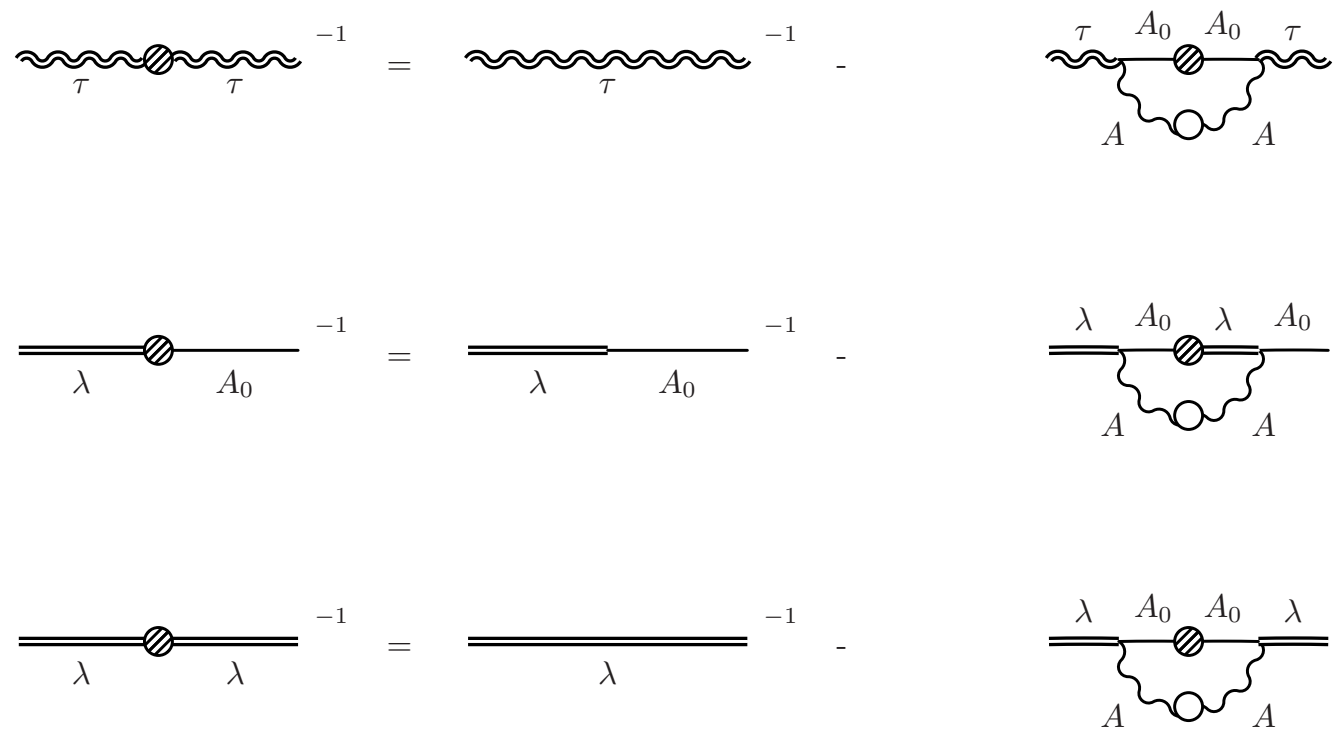

Figure 3. Diagrams for the relevant ISDEs. The five different types of lines are explained in Fig. 2. As usual, shaded circles represent the instantaneous, full propagator and the empty circles are non-instantaneous. 


\section{Conclusion}

We have a local, renormalizable quantum field theory with the following interesting properties:

- It provides a cut-off at the Gribov horizon.

- The Kugo-Ojima color confinement condition is satisfied.

- The vacuum is a perfect dielectric.

- The Maggiore-Schaden shift provides a BRST-invariant Lagangian.

- BRST-symmetry is spontaneously broken, but perhaps only in the unphysical sector.

- In Coulomb gauge, the color-Coulomb potential rises linearly or super-linearly when the Wilson potential is linearly rising.

- In the ISDE, all loops consist of one instantaneous propagator and the equal-time part of a noninstantaneous propagator.

\section{References}

[1] V.N. Gribov, Quantization of non-Abelian Gauge Theories, Nucl. Phys. B 139, 1 (1978).

[2] Daniel Zwanziger, No Confinement without Coulomb Confinement, Phys. Rev. Letts, 90, 102001 (2003) and arXiv:hep-lat/0209105.

[3] Daniel Zwanziger, Renormalization in the Coulomb gauge and order parameter for confinement in QCD, Nucl. Phys. B 518 237-272 (1998).

[4] Daniel Zwanziger, Local and Renormalizable Action from the Gribov Horizon, Nucl. Phys. B323, 513 (1989).

[5] N. Maggiore, M. Schaden, Landau gauge within the Gribov horizon, Phys. Rev. D50, 6616 (1994).

[6] M. Schaden, habilitation thesis on Kovariante Eichfixierung und Dynamische Brechung der BRS Symmetrie von Yang-Mills Eichtheorien, Technische Universität München, Germany (1996).

[7] Martin Schaden and Daniel Zwanziger, Phys. Rev. D92, 025001 (2015), and arXiv:1412.4823 [hep-ph].

[8] Martin Schaden and Daniel Zwanziger, Living with spontaneously broken BRST symmetry. II. Poincaré invariance, Phys. Rev. D92, 025002 (2015) and arXiv:1501.05974 [hep-th].

[9] Alkofer, Reinhard and Maas, Axel and Zwanziger, Daniel, Truncating first-order DysonSchwinger equations in Coulomb-Gauge Yang-Mills theory, Few Body Syst, 47, 73 (2010) and arXiv:0905.4594.

[10] T. Kugo, I. Ojima, Prog. Theor. Phys. Suppl.66, 1 (1979) ;Erratum Prog. Theor. Phys.71, 1121 (1984).

[11] T. Kugo, The Universal renormalization factors $Z(1) / Z(3)$ and color confinement condition in nonAbelian gauge theory, arXiv:hep-th/9511033.

[12] H. Reinhardt, Phys. Rev. Lett. 101, 061602 (2008), 08030504.

[13] N. Vandersickel, D. Zwanziger, The Gribov problem and QCD dynamics, Phys. Rept.520, 175 (2012) and arXiv:1202.1491 [hep-th].

[14] R.F. Sobreira, S.P. Sorella, Introduction to the Gribov Ambiguities In Euclidean Yang-Mills Theories, arXiv:hep-th/0504095.

[15] A. Andrasi, J. C. Taylor, The effective action in QCD, Annals of Physics 351 407-417 (2014) and arXiv:1406.7802 [hep-th], and references found there.

[16] Patrick Cooper and Daniel Zwanziger, Local QCD Action at Finite Temperature, Phys. Rev. D 93, 105026 (2016) and arXiv:1512.08858.

[17] Patrick Cooper and Daniel Zwanziger (in preparation). 